\title{
The Critical Coagulation Concentration of Counterions: Spherical Particles in Asymmetric Electrolyte Solutions
}

\author{
JyH-Ping Hsu ${ }^{1}$ AND Yung-ChIH KuO \\ Department of Chemical Engineering, National Taiwan University, Taipei, Taiwan 10617, Republic of China
}

Received June 12, 1996; accepted September 3, 1996

\begin{abstract}
The ratio of the critical coagulation concentration (CCC) of counterions is evaluated for spherical particles and asymmetric electrolytes. A perturbation method is adopted to solve the Poisson- Boltzmann equation governing the electrical potential distribution of the system under consideration. On the basis of the result obtained, an approximate expression for the C C C is derived. Another approach based on the Derjaguin approximation is also used to estimate the C CC. We show that the C C C ratio of counterions is a complicated function of the valences of the ion species in the liquid phase and the sizes of particles. $D$ epending upon the thickness of the Debye length, the CCC ratio of counterions for various combinations of electrolytes can be estimated. The classic Schulze- $\mathrm{H}$ ardy rule for planar particles in a symmetric electrolyte solution can be recovered as a limiting case of the present model. If the surface potential is low, the effect of curvature on the C CC ratio of counterions is negligible. 1997 Academic Press

Key words: critical coagulation concentration, counterions, ratio; particles, charged, spherical; electrolyte solutions, asymmetric; interaction energy, Derjaguin approximation; Poisson- Boltzmann equation, perturbation solution.
\end{abstract}

\section{INTRODUCTION}

The minimum concentration of electrolyte required to induce the coagulation of a stable colloidal suspension is defined as the critical coagulation concentration (CCC). The $\mathrm{CCC}$ of counterions is found to be inversely proportional to the sixth power of its valence, the so-called Schulze-Hardy rule. For counterions of valences 3,2 , and 1, this rule suggests that the CCC ratio is $3^{-6}: 2^{-6}: 1^{-6}$, or roughly, $1: 11: 729$. The Schulze-Hardy rule can be interpreted theoretically by the DLVO theory (1), which considers the electrostatic repulsion force and the van der Waals attraction force between two interacting particles. The key assumptions made in the derivation of this rule are planar surfaces, symmetric electrolytes, and a high level of surface potential (1). Although some of these assumptions are violated obviously (2) and

\footnotetext{
${ }^{1}$ To whom correspondence should be addressed.
}

deviations in the experimental observations from the theoretical predictions are not uncommon $(3,4)$, the SchulzeHardy rule is still widely adopted in practice for its convenience.

The difficulty of modifying the Schulze-Hardy rule to a general case depends largely on the types of electrolyte and the shapes of a particle. These factors are closely related to the resolution of the Poisson-Boltzmann equation governing the electrostatic potential distribution around a charged surface (5-9). The solution to this equation is essential to the evaluation of the electrostatic repulsion force between two particles. Since the exact analytical solution to the PoissonBoltzmann equation under a general condition has not been reported, the corresponding CCC is unknown. In a recent study, an attempt was made to take the asymmetry of electrolytes into account for planar particles. It was found that for a common monovalent anion, the CCC ratio of counterions for valences 3,2 , and 1 is roughly proportional to the -6.35 power of the counterion valence (10).

In the present study, the Schulze-Hardy rule is extended to the case of spherical surfaces in an arbitrary $a: b$ electrolyte solution. In other words, the effect of the curvature of dispersed entities on the CCC ratio of counterions is investigated.

\section{MODELING}

The analysis is begun by considering a charged spherical particle immersed in an $a: b$ electrolyte solution. Without loss of generality, we assume that the particle is negatively charged. The electrical potential distribution around the particle is described by the Poisson-Boltzmann equation (9)

$$
\frac{d^{2} \psi}{d X^{2}}+\frac{2}{X} \frac{d \psi}{d X}=\frac{\exp (b \psi)-\exp (-a \psi)}{a+b}
$$

where $\psi=e \phi /\left(k_{B} T\right), X=\kappa r, \kappa^{2}=e^{2} a(a+b) n_{a}^{0} /\left(\epsilon_{0} \epsilon_{r} k_{B} T\right)$. Here $\phi$ is the electrical potential, $e$ denotes the elementary charge, $\kappa$ is the reciprocal Debye length, $\epsilon_{r}$ and $\epsilon_{0}$ are the 
relative permittivity of solution and the permittivity of the vacuum, respectively, $n_{a}^{0}$ is the number concentration of cation in the bulk liquid phase, $k_{B}$ and $T$ are, respectively, the Boltzmann constant and the absolute temperature, and $r$ is the distance measured from the center of the particle. The boundary conditions associated with Eq. [1] are assumed to be

$$
\begin{gathered}
\psi \rightarrow 0 \text { and }(d \psi / d X) \rightarrow 0 \text { as } X \rightarrow \infty \\
\psi=\psi_{0} \text { at } X=X_{0}
\end{gathered}
$$

where $\psi_{0}$ is the dimensionless surface potential and $X_{0}$ is the dimensionless radius of the particle, $X_{0}=\kappa r_{0}, r_{0}$ being the radius. For a sufficiently large $X_{0}$, an approximate solution to Eq. [1] subject to Eqs. [1a] and [1b] is (11)

$$
\psi \cong h_{0}+\frac{h_{1}}{X_{0}}+\frac{h_{2}}{X_{0}^{2}}
$$

where

$$
\begin{gathered}
\tanh \left(a h_{0} / 4\right)=\left[\tanh \left(a \psi_{0} / 4\right)\right] \exp \left[-k_{3}\left(X-X_{0}\right)\right] \\
h_{1}=\left[1 /\left(a k_{3}\right)\right] \sinh \left(a h_{0} / 2\right)\left\{\tanh ^{2}\left(a \psi_{0} / 4\right)\right. \\
\left.-\tanh ^{2}\left(a h_{0} / 4\right)-2 \ln \left[\tanh \left(a \psi_{0} / 4\right) / \tanh \left(a h_{0} / 4\right)\right]\right\}, \\
h_{2}=\frac{a}{2 k_{3}} \sinh \left(a h_{0} / 2\right) \int_{\psi_{0}}^{h_{0}} \frac{1}{\sinh ^{2}\left(a h_{0} / 2\right)}\left\{\frac{a k_{3}}{4} h_{1}^{2} \sinh \left(a h_{0} / 2\right)\right. \\
+\frac{2 h_{1}}{1+\cosh \left(a h_{0} / 2\right)}-\frac{4}{a}\left[\tanh \left(a h_{0} / 4\right)\right]\left[X\left(h_{0}\right)-X_{0}\right] \\
\left.-\frac{4 \tanh ^{2}\left(a h_{0} / 4\right)-8 \ln \left[\cosh \left(a h_{0} / 4\right)\right]}{a k_{3} \sinh \left(a h_{0} / 2\right)}\right\} d h_{0}
\end{gathered}
$$

with

$$
\begin{array}{r}
k_{3}= \begin{cases}{\left[(k-2) k_{1}+2 k_{2}\right] / k,} & \text { if } k \leqslant 4 \\
{\left[2 k_{1}+(k-2) k_{2}\right] / k,} & \text { if } k>4\end{cases} \\
X\left(h_{0}\right)=\frac{1}{2 k_{3}} \ln \left[\left(\frac{\cosh \left(a h_{0} / 2\right)+1}{\cosh \left(a h_{0} / 2\right)-1}\right)\right. \\
\left.\times\left(\frac{\cosh \left(a \psi_{0} / 2\right)-1}{\cosh \left(a \psi_{0} / 2\right)+1}\right)\right]+X_{0}
\end{array}
$$

In these expressions, $k_{1}=2 /\left\{k^{1 / 2}\left[(k / 2)^{2 /(k-2)}-1\right]\right\}, k_{2}=2 /$ $k^{1 / 2}$, and $k=2+2 b / a(7)$.

The electrostatic free energy $F_{e l}^{t}$, for a charged system can be expressed by (11)

$$
F_{e l}^{t}=-\left(\epsilon_{0} \epsilon_{r} / 2\right) \int \phi \overrightarrow{\mathrm{E}} \cdot \overrightarrow{\mathrm{n}} d A
$$

where $d A$ is a differential area, $\vec{E}$ is the strength of electric field, and $\vec{n}$ is the unit outer normal vector. For a system containing $K$ particles Eq. [3] gives

$$
F_{e l}^{t}=\sum_{n=1}^{K}\left[\left(\sigma_{n} / 2\right) \int_{n}\left(\sum_{\substack{n^{\prime}=1 \\ n^{\prime} \neq n}}^{K} \phi_{n^{\prime}}\right) d A_{n}\right]
$$

where the subscripts $n$ and $n^{\prime}$ denote the $n$th and the $n^{\prime}$ th particles, respectively, and $\sigma_{n}$ is the surface charge density of the $n$th particle. Here, we assume that the distance between any two particles is on the order of the Debye length. The electroneutrality requires that

$$
\sigma_{n}=\frac{-1}{4 \pi r_{0, n}^{2}} \int_{r_{0, n}}^{\infty} 4 \pi r_{n}^{2} \rho_{n} d r_{n}
$$

where $r_{n}$ is the distance measured from the center of the $n$th particle, $r_{0, n}$ is its radius, and $\rho_{n}$ is the space charge density for the $n$th isolated particle. The electrical interaction energy $I_{e l}$ can be estimated by

$$
I_{e l}(\underline{\mathrm{L}})=F_{e l}^{t}(\underline{\mathrm{L}})-F_{e l}^{t}(\infty)
$$

where $\mathrm{L}$ is the set of distance $L_{n, n^{\prime}}, n, n^{\prime}=1,2, \ldots, K, n$ $\neq n^{\prime}, L_{n, n}$ being the closest dimensionless surface-to-surface distance between particles $n$ and $n^{\prime}$ with $L_{n, n^{\prime}}=L_{n^{\prime}, n}$, and $F_{e l}^{t}(\infty)$ is the value of $F_{e l}^{t}$ when any pair of particles is infinitely apart. For two interacting particles Eqs. [4] and [6] lead to

$$
I_{e l}(L)=\left(\sigma_{1} / 2\right) \int_{1} \phi_{2} d A_{1}+\left(\sigma_{2} / 2\right) \int_{2} \phi_{1} d A_{2}
$$

where $L=\kappa L_{0}, L_{0}$ being the closest surface-to-surface distance between two particles.

Based on Eqs. [2a]-[2c], if $X_{0}$ is sufficiently large, the potential distribution for a large $\left(X_{n}-X_{0, n}\right)=\kappa\left(r_{n}-r_{n, 0}\right)$ can be approximated by

$$
\psi_{n} \cong\left(A_{n}+B_{n} X_{n}\right) \exp \left(-k_{3} X_{n}\right)
$$

where $\psi_{n}=e \phi_{n} / k_{B} T$, and

$$
A_{n}=\left\{\frac{8}{a}\left[\tanh \left(\frac{a \psi_{0, n}}{4}\right)\right]+\frac{2}{a k_{3} X_{0, n}}\left[\tanh \left(\frac{a \psi_{0, n}}{4}\right)\right]^{3}\right\} e^{k_{3} X_{0, n}}
$$




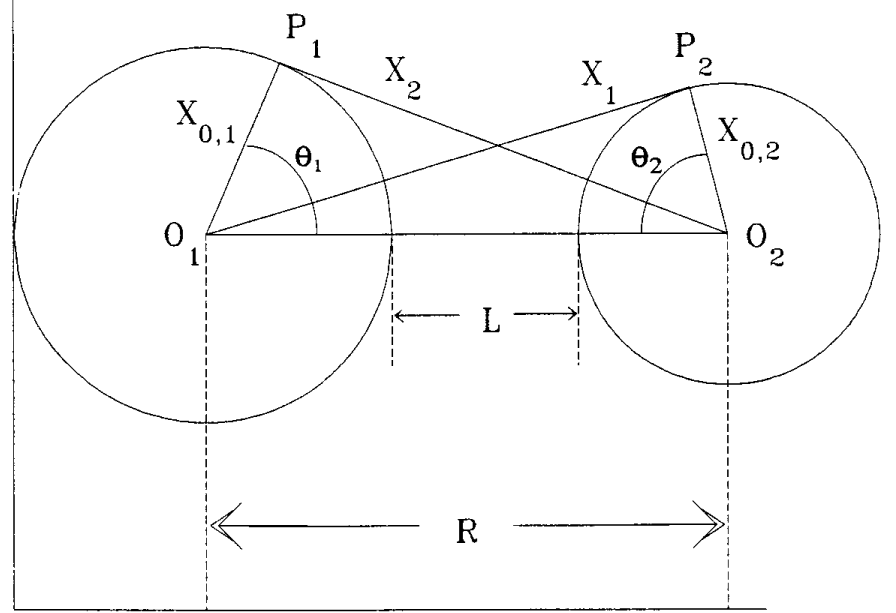

FIG . 1. Schematic representation of two interacting particles. $X_{1}, X_{2}, L$, and $R$ are the dimensionless distances; $X_{0,1}$ and $X_{0,2}$ are the dimensionless radii of particles 1 and 2 , respectively; $\theta_{1}$ is the angle defined by the line segments $O_{1}-O_{2}$ and $O_{1}-P_{1}$; and $\theta_{2}$ is the angle defined by the line segments $\mathrm{O}_{2}-\mathrm{O}_{1}$ and $\mathrm{O}_{2}-\mathrm{P}_{2}$.

$$
B_{n}=\left\{\frac{-4}{a X_{0, n}}\left[\tanh \left(\frac{a \psi_{0, n}}{4}\right)\right]\right\} e^{k_{3} X_{0, n}}
$$

By referring to Fig. 1, the differential area $d A_{n}$ can be expressed as

$$
d A_{n}=2 \pi \kappa^{-2} X_{0, n}^{2} \sin \theta_{n} d \theta_{n}
$$

where $\theta_{n}$ is the angle between line segment $O_{1}-O_{2}(R)$ and $O_{n}-P_{n}\left(X_{0, n}\right), R$ being the dimensionless center-to-center distance between two particles. We have

$$
X_{n^{\prime}}^{2}=R^{2}+X_{0, n}^{2}-2 R X_{0, n} \cos \theta_{n}, n^{\prime} \neq n
$$

Substituting Eqs. [8]-[10] into Eq. [7] and conducting the integration, we obtain

$$
I_{e l}(L) \cong \sum_{n=1}^{2} G_{n}\left(C_{n^{\prime}} L+D_{n^{\prime}}\right) e^{-k_{3} L}, n^{\prime} \neq n
$$

where

$$
C_{n^{\prime}}=-4\left[\tanh \left(\frac{a \psi_{0, n^{\prime}}}{4}\right)\right] /\left(a \mathrm{X}_{0, n^{\prime}}\right)
$$

$$
\begin{aligned}
D_{n^{\prime}}= & 2\left[\tanh \left(\frac{a \psi_{0, n^{\prime}}}{4}\right)\right]\left\{\left\{4 k_{3} X_{0, n}\right.\right. \\
& \left.+\left[\tanh \left(\frac{a \psi_{0, n^{\prime}}}{4}\right)\right]^{2}-4\right\} /\left(a k_{3} X_{0, n^{\prime}}\right)+\frac{4}{a} \\
& +\left\{-2 k_{3}^{2} X_{0, n}^{2}+4 k_{3} X_{0, n}+\left(1-k_{3} X_{0, n}\right)\right. \\
& \left.\times\left[\tanh \left(\frac{a \psi_{0, n^{\prime}}}{4}\right)\right]^{2}-4\right\} /\left[a k_{3}^{2} X_{0, n^{\prime}}\left(X_{0, n}+X_{0, n^{\prime}}\right)\right] \\
& \left.+4\left(1-k_{3} X_{0, n}\right) /\left[a k_{3}\left(X_{0, n}+X_{0, n^{\prime}}\right)\right]\right\} \\
& +C_{n^{\prime}}\left(X_{0, n}+X_{0, n^{\prime}}\right), n^{\prime} \neq n
\end{aligned}
$$

For illustration, we consider two identical particles in the following discussion. In this case, the subscripts $n$ and $n^{\prime}$ can be dropped.

For a sufficiently small $L / X_{0}$, the van der Waals potential $I_{v d w}$ can be estimated by (1)

$$
I_{v d w}(L)=-A_{132} X_{0} / 12 L
$$

where $A_{132}$ is the Hamaker constant. The total interaction energy $I_{t}(L)$ is the sum of $I_{e l}(L)$ and $I_{v d w}(L)$, i.e.,

$$
I_{t}(L)=I_{e l}(L)+I_{v d w}(L)
$$

At the CCC,

$$
I_{t}=0 \text { and } d I_{t} / d L=0
$$

Let the value of $L$ at the CCC be $L_{c}$. Substituting Eqs. [11][13] into Eq. [14], we obtain

$$
\begin{aligned}
2 G\left(C L_{c}+D\right) \exp \left(-k_{3} L_{c}\right)-\frac{A_{132} X_{0}}{12 L_{c}}=0 \\
-2 k_{3} G\left(C L_{c}+D\right) \exp \left(-k_{3} L_{c}\right) \\
+2 G C \exp \left(-k_{3} L_{c}\right)+\frac{A_{132} X_{0}}{12 L_{c}^{2}}=0
\end{aligned}
$$

These expressions yield

$$
L_{c}^{2}-\left(\frac{2}{k_{3}}-\frac{D}{C}\right) L_{c}-\frac{D}{k_{3} C}=0
$$


Since $D / C$ is large for the present system ( $X_{0}$ is large), we have

$$
L_{c} \cong \frac{1}{k_{3}}\left\{1-2 /\left\{k_{3} X_{0}+4-\left[\tanh \left(\frac{a \psi_{0}}{4}\right)\right]^{2} / 2\right\}\right\} .
$$

If $\psi_{0} \rightarrow-\infty$, Eq. [5] leads to (Appendix A)

$$
\sigma \propto a n_{a}^{0} /\left(k_{3} \kappa\right)
$$

Substituting Eqs. [17] and [18] into Eq. [15a] yields

$$
\begin{aligned}
& \frac{a n_{a, c}^{0}}{k_{3}^{3} \kappa^{3}}\left[\frac{-2}{a}+\frac{3}{a k_{3} X_{0}}\right.\left.-\frac{8}{a k_{3} X_{0}\left(k_{3} X_{0}+7 / 2\right)}\right] \\
& \times\left(1-\frac{2}{k_{3} X_{0}+7 / 2}\right)^{2}=\text { constant }
\end{aligned}
$$

where $n_{a, c}^{0}$ denotes the CCC of cations (counterions). This expression implies that

$n_{a, c}^{0} \propto \frac{1}{a^{3}(a+b)^{3} k_{3}^{6}}\left[1-\frac{3}{k_{3} X_{0}}-\frac{8}{k_{3} X_{0}+7 / 2}\left(1-\frac{1}{k_{3} X_{0}}\right)\right]$

On the other hand, if $\psi_{0} \rightarrow 0$, it can be shown that (Appen$\operatorname{dix} \mathrm{A})$

$$
\sigma \propto\left[a(a+b) k_{3} e n_{a}^{0} \psi_{0} \kappa^{-1}\right]\left[1+\left(1+a^{2} \psi_{0}^{2} / 32\right) / k_{3} X_{0}\right]
$$

Substituting Eqs. [17] and [21] into Eq. [15a] yields

$$
n_{a, c}^{0} \propto \frac{\psi_{0}^{4}}{a(a+b) k_{3}^{2}}\left[1-\frac{2}{k_{3} X_{0}}-\frac{8}{k_{3} X_{0}+4}\left(1-\frac{1}{k_{3} X_{0}}\right)\right]
$$

\section{Another Approach}

If the Derjaguin approximation (12) is applicable, the electrical potential energy between two particles $V_{e l}$ can be estimated by

$$
V_{e l}=\frac{\pi X_{0}}{\kappa^{2}} \int_{0}^{2 X_{0}}\left(1-\frac{L_{1}}{2 X_{0}}\right) V_{R} d L_{1}
$$

where

$$
L_{1}=L_{2}-L
$$

In these expressions, $L_{2}=\kappa L_{3}, L_{3}$ is the surface-to-surface distance between two particles, $L$ is defined under Eq. [7], and $V_{R}$ is the electrical potential energy for two planar parallel surfaces.

The electrical potential energy $V_{R}$ can be calculated by (10)

$$
\begin{aligned}
V_{R} & =\kappa^{-1} \int_{L}^{\infty} F_{R} d L \\
& =\frac{32(a+b) n_{a}^{0} k_{B} T}{a k_{3} \kappa}\left[\tanh ^{2}\left(a \psi_{0} / 4\right)\right] \exp \left(-k_{3} L\right)
\end{aligned}
$$

Substituting Eq. [24] into Eq. [23] yields

$$
\begin{array}{r}
V_{e l}=\frac{32(a+b) \pi X_{0} n_{a}^{0} k_{B} \mathrm{~T}}{a k_{3}^{2} \kappa^{3}}\left[\tanh ^{2}\left(a \psi_{0} / 4\right)\right] \exp \left(-k_{3} L_{0}\right) \\
\times\left[1-\frac{1}{2 k_{3} X_{0}}\left(1-\exp \left(-2 k_{3} X_{0}\right)\right)\right]
\end{array}
$$

The van der Waals potential, the total interaction energy, and the criterion for CCC are described by Eqs. [12]-[14], respectively. Let $L_{c}$ be the value of $L$ at which coagulation occurs. Then, Eqs. [12]-[14] and [25] lead to $L_{c}=1 / k_{3}$. Substituting this $L_{c}$, Eqs. [12] and [25] into Eq. [13], applying Eq. [14], and solving the resultant expression for $n_{a, c}^{0}$, the CCC of cations, we obtain

$$
n_{a, c}^{0}=\frac{\lambda \tanh ^{4}\left(a \psi_{0} / 4\right)}{a^{5}(a+b) k_{3}^{6}} \cdot \frac{\left(4 \pi \epsilon_{0} \epsilon_{r}\right)^{3}\left(k_{B} T\right)^{5}(48)^{2}}{e^{6} A_{123}^{2} \pi(\exp 2)}
$$

where

$$
\lambda=\left[1-\frac{1}{2 k_{3} X_{0}}\left(1-\exp \left(-2 k_{3} X_{0}\right)\right)\right]^{2}
$$

On the basis of these expressions, the CCC of counterions can be estimated.

If $\psi_{0} \rightarrow-\infty$, Eq. [26] gives

$$
n_{a, c}^{0} \propto \frac{\lambda}{a^{5}(a+b) k_{3}^{6}}
$$

For a sufficiently large $X_{0}, \lambda \rightarrow 1$, and Eq. [27] leads to the result for planar particles (10). Furthermore, if the electrolyte is symmetric, $a=b=v$, then

$$
n_{a, c}^{0} \propto 1 / v^{6}
$$

This is the Schulze-Hardy rule. 
TABLE 1

Variation in the Ratio $\left[n_{a, c, a b}^{0} / n_{a, c, 3: 3}^{0}\left(X_{0} \rightarrow \infty\right)\right]$ as a Function of $X_{0}$ and the Types of Electrolyte for $\Psi_{0} \rightarrow-\infty$

\begin{tabular}{|c|c|c|c|c|c|c|c|}
\hline$a: b$ & $X_{0}$ & 200 & 100 & 80 & 50 & 30 & 20 \\
\hline $3: 3$ & 1.000 & 0.945 & 0.893 & 0.868 & 0.793 & 0.699 & 0.527 \\
\hline $3: 2$ & 1.384 & 1.311 & 1.241 & 1.207 & 1.108 & 0.941 & 0.749 \\
\hline 3:1 & 1.522 & 1.449 & 1.379 & 1.345 & 1.244 & 1.075 & 0.880 \\
\hline $2: 3$ & 7.379 & 6.964 & 6.562 & 6.366 & 5.798 & 4.850 & 3.766 \\
\hline $2: 2$ & 11.391 & 10.774 & 10.177 & 9.886 & 9.038 & 7.622 & 5.998 \\
\hline $2: 1$ & 17.232 & 16.365 & 15.525 & 15.113 & 13.915 & 11.904 & 9.586 \\
\hline $1: 3$ & 276.161 & 258.242 & 240.998 & 232.618 & 208.382 & 168.368 & 123.157 \\
\hline $1: 2$ & 365.385 & 343.833 & 323.022 & 312.882 & 283.464 & 234.590 & 178.920 \\
\hline 1:1 & 729.000 & 689.550 & 651.346 & 632.691 & 578.431 & 487.813 & 383.888 \\
\hline
\end{tabular}

On the other hand, if $\psi_{0} \rightarrow 0$, Eq. [26] leads to

$$
n_{a, c}^{0} \propto \frac{\lambda}{a(a+b) k_{3}^{6}}
$$

For a large $X_{0}$ this expression becomes the result for planar particles. Furthermore, if $a=b=v$, then

$$
n_{a, c}^{0} \propto 1 / v^{2}
$$

This is consistent with the result for planar particles (10).

\section{RESULTS AND DISCUSSION}

The variation in the ratio $\left[n_{a, c, a: b} / n_{a, c, 3: 3}\left(X_{0} \rightarrow \infty\right)\right]$ as a function of $X_{0}$ and various types of electrolyte for $\psi_{0} \rightarrow-\infty$ is shown in Table 1 . Note that as $X_{0} \rightarrow \infty$, the CCC ratio of cations for symmetric electrolytes is 1:11.391:729, the same as that predicted by the Schulze-Hardy rule.

TABLE 2

Approximate C C C Ratio of Counterions at Various Assumed $X_{0}$ $R$ atios and Types of Electrolyte for $\Psi_{0} \rightarrow-\infty$

\begin{tabular}{llccc}
\hline & \multicolumn{4}{c}{ Types of electrolyte } \\
\cline { 2 - 5 } $\begin{array}{c}\text { Approximate } \\
X_{0} \text { Ratio }\end{array}$ & \multicolumn{1}{c}{$S$} & $A_{1}$ & $A_{2}$ & $A_{3}$ \\
\hline $20: 50: 200$ & $1: 17.1: 1038$ & $1: 15.8: 784$ & $1: 12.1: 459$ & $1: 11.0: 490$ \\
$30: 80: \infty$ & $1: 14.8: 1090$ & $1: 14.1: 678$ & $1: 10.5: 388$ & $1: 9.5: 413$ \\
$50: 100: \infty$ & $1: 12.8: 919$ & $1: 12.5: 586$ & $1: 9.2: 330$ & $1: 8.3: 348$ \\
$100: 200: \infty$ & $1: 12.1: 816$ & $1: 11.9: 529$ & $1: 8.7: 294$ & $1: 7.8: 309$ \\
$200: \infty: \infty$ & $1: 12.1: 771$ & $1: 11.9: 503$ & $1: 8.7: 279$ & $1: 7.8: 292$ \\
$\infty: \infty: \infty$ & $1: 11.4: 729$ & $1: 11.3: 479$ & $1: 8.2: 264$ & $1: 7.4: 276$ \\
\hline
\end{tabular}

Note. Type $S$ denotes symmetric electrolytes, the $X_{0}$ ratio is $X_{0,3: 3}: X_{0,2: 2}: X_{0,1: 1}$, and the $n_{a, c}^{0}$ ratio is $n_{a, c, 3: 3}^{0}: n_{a, c, 2: 2}^{0}: n_{a, c, 1: 1}^{0}$. Type $A_{\mathrm{b}}$ denotes asymmetric electrolytes, the valence of anion is $b$, the $X_{0}$ ratio is $X_{0,3: b}: X_{0,2: b}: X_{0,1: b}$, and the $n_{a, c}^{0}$ ratio is $n_{a, c, 3: 3}^{0}: n_{a, c, 2: 2}^{0}: n_{a, c, 1: 1}^{0}$.
According to Eqs. [20] and [22], the CCC of counterions is related to the type of electrolyte $(a: b)$, and the dimensionless size of a particle $X_{0}\left(=\kappa r_{0}\right)$. For a fixed particle size $r_{0}$, the latter is a function of the Debye length, which is related to the concentrations of ions. This means that the CCC ratio of counterions needs to be determined through an iterative procedure; the algorithm presented in Appendix B is suggested.

Table 2 illustrates the CCC ratio of counterions at several assumed $X_{0}$ ratios and types of electrolyte for $\psi_{0} \rightarrow$ $-\infty$. The result shown in this table reveals that, depending upon the $\mathrm{X}_{0}$ ratio, the $\mathrm{CCC}$ ratio of counterions can take various values.

The variation in the ratio $\left[n_{a, c, a: b} / n_{a, c, 3: 3}\left(X_{0} \rightarrow \infty\right)\right]$ at two levels of $X_{0}$ and various types of electrolyte for the case $\psi_{0} \rightarrow 0$ is shown in Table 3 , and the corresponding CCC ratio of counterions is illustrated in Table 4. If $\psi_{0}$ is low, $X_{0}$ is about the same for each type of CCC ratio. As suggested by Table 4, the CCC ratio for spherical particles is close to that for planar particles. In other words, if the surface potential is low, the effect of curvature on the CCC ratio of counterions is negligible.

TABLE 3

Variation in the Ratio $\left[n_{a, c, a b b}^{0} / n_{a, c, 3: 3}^{0}\left(X_{0} \rightarrow \infty\right)\right]$ as a Function

\begin{tabular}{|c|c|c|}
\hline$a: b$ & $X_{0}$ & 20 \\
\hline $3: 3$ & 1.000 & 0.583 \\
\hline $3: 2$ & 1.114 & 0.664 \\
\hline $3: 1$ & 1.150 & 0.722 \\
\hline $2: 3$ & 1.947 & 1.109 \\
\hline $2: 2$ & 2.250 & 1.313 \\
\hline $2: 1$ & 2.583 & 1.572 \\
\hline $1: 3$ & 6.512 & 3.355 \\
\hline $1: 2$ & 7.149 & 3.946 \\
\hline $1: 1$ & 9.000 & 5.250 \\
\hline
\end{tabular}
of $X_{0}$ and the Types of Electrolyte for $\Psi_{0} \rightarrow 0$ 
TABLE 4

Approximate CCC Ratio of Counterions at Various Assumed $X_{0}$ $R$ atios and Types of Electrolyte for $\Psi_{0} \rightarrow 0$

\begin{tabular}{lcccc}
\hline & \multicolumn{4}{c}{ Types of electrolyte } \\
\cline { 2 - 5 } $\begin{array}{c}\text { Approximate } \\
X_{0} \text { ratio }\end{array}$ & $S$ & $A_{1}$ & $A_{2}$ & $A_{3}$ \\
\hline $20: 20: 20$ & $1: 2.25: 9.00$ & $1: 2.18: 7.27$ & $1: 1.98: 5.94$ & $1: 1.90: 5.75$ \\
$\infty: \infty: \infty$ & $1: 2.25: 9.00$ & $1: 2.25: 7.83$ & $1: 2.02: 6.42$ & $1: 1.95: 6.51$ \\
\hline
\end{tabular}

Note. The symbols are the same as those used in Table 2.

If $\psi_{0} \rightarrow-\infty$ and the electrolyte is symmetric, $a=b=v$, and $k_{3}=1$, Eq. [20] becomes

$$
n_{a, c}^{0} \propto \frac{1}{v^{6}}\left[1-\frac{3}{X_{0}}-\frac{8}{X_{0}+7 / 2}\left(1-\frac{1}{X_{0}}\right)\right]
$$

Furthermore, if $X_{0} \rightarrow \infty$, a particle can be treated as a flat surface, Eq. [29] reduces to Eq. [27a].

On the other hand, if $\psi_{0} \rightarrow 0$ and the electrolyte is symmetric, Eq. [22] gives

$$
n_{a, c}^{0} \propto \frac{\psi_{0}^{4}}{v^{2}}\left[1-\frac{2}{X_{0}}-\frac{8}{X_{0}+4}\left(1-\frac{1}{X_{0}}\right)\right]
$$

If $X_{0} \rightarrow \infty$, then Eq. [28a] is recovered.

\section{Derjaguin Approximation}

The variation in the ratio $\left[n_{a, c, a: b} / n_{a, c, 3: 3}\left(\psi_{0} \rightarrow-\infty\right)\right]$ for various values of $X_{0}$ and types of electrolyte for the case $\psi_{0} \rightarrow$ $-\infty$ based on the Derjaguin approximation is shown in Table 5. According to Eqs. [27] and [28], the CCC ratio of counterions is a function of the type of electrolyte and the dimensionless size of particle. The algorithm presented in Appendix B can also be employed.

\section{TABLE 5}

Variation in the Ratio $\left[n_{\mathrm{a}, c, a \mathrm{a}, \mathrm{l}} / \boldsymbol{n}_{a, c, 3: 3}\left(X_{0} \rightarrow \infty\right)\right]$ as a Function of $X_{0}$ and the Types of Electrolyte Solution Based on the D erjaguin

\begin{tabular}{|c|c|c|c|c|c|}
\hline$a: b$ & $X_{0}$ & $\infty$ & 65 & 25 & 10 \\
\hline $3: 3$ & & 1.000 & 0.985 & 0.960 & 0.903 \\
\hline $3: 2$ & & 0.961 & 0.947 & 0.924 & 0.870 \\
\hline $3: 1$ & & 0.676 & 0.667 & 0.653 & 0.618 \\
\hline $2: 3$ & & 11.529 & 11.345 & 11.054 & 10.361 \\
\hline $2: 2$ & & 11.391 & 11.216 & 10.940 & 10.280 \\
\hline $2: 1$ & & 9.693 & 9.555 & 9.336 & 8.814 \\
\hline $1: 3$ & & 1104.646 & 1084.296 & 1052.131 & 975.757 \\
\hline $1: 2$ & & 822.117 & 808.369 & 786.613 & 734.826 \\
\hline $1: 1$ & & 729.000 & 717.828 & 700.132 & 657.923 \\
\hline
\end{tabular}
Approximation for $\Psi_{0} \rightarrow-\infty$
TABLE 6

Approximate CCC R atio of Counterions at Various Assumed $X_{0} R$ atios and the Types of Electrolyte Based on the Derjaguin

\begin{tabular}{|c|c|c|c|c|c|}
\hline Type & $X_{0}$ Ratio & $10: 25: \infty$ & $25: 65: \infty$ & $65: \infty: \infty$ & $\infty: \infty: \infty$ \\
\hline Type $S$ & & 1:12.1:807 & 1:11.7:759 & 1:11.6:740 & 1:11.4:729 \\
\hline Type $A_{1}$ & & 1:15.1:1180 & 1:14.6:1116 & 1:14.5:1093 & 1:14.3:1078 \\
\hline Type $A_{2}$ & & 1:12.6:945 & 1:12.1:890 & 1:12.0:868 & 1:11.8:855 \\
\hline Type $A_{3}$ & & 1:12.2:1223 & 1:11.8:1151 & 1:11.7:1121 & 1:11.5:1105 \\
\hline
\end{tabular}
Approximation for $\Psi_{0} \rightarrow-\infty$

Note. The symbols are the same as those used in Table 2.

Table 6 illustrates the CCC ratio of cations at several assumed $X_{0}$ ratios and types of electrolyte for $\psi_{0} \rightarrow-\infty$. The result shown in this table reveals that the CCC ratio of counterions is a function of the types of electrolyte and the particle size.

The variation in the ratio $\left[n_{a, c, a: b} / n_{a, c, 3: 3}\left(X_{0} \rightarrow \infty\right)\right]$ at two levels of $X_{0}$ and various types of electrolyte for the case $\psi_{0}$ $\rightarrow 0$ is shown in Table 7 . The CCC ratio of counterions for various $X_{0}$ ratios is about the same. The result is

$$
\begin{aligned}
& n_{a, c, 3: 3}: n_{a, c, 2: 2}: n_{a, c, 1: 1}=1: 2.25: 9.0 \\
& n_{a, c, 3: 1}: n_{a, c, 2: 1}: n_{a, c, 1: 1}=1: 2.8: 13.2 \\
& n_{a, c, 3: 2}: n_{a, c, 2: 2}: n_{a, c, 1: 2}=1: 2.3: 10.5 \\
& n_{a, c, 3: 1}: n_{a, c, 2: 1}: n_{a, c, 1: 1}=1: 2.3: 13.5
\end{aligned}
$$

In this case, the CCC ratio for spherical particles is insensitive to curvature, similar to the results of Table 4.

The result based on the Derjaguin approximation is readily applicable to a suspension of spherical particles coated with

\begin{tabular}{|c|c|c|}
\hline$a: b$ & $X_{0}$ & 10 \\
\hline $3: 3$ & 1.000 & 0.903 \\
\hline $3: 2$ & 0.961 & 0.870 \\
\hline $3: 1$ & 0.676 & 0.618 \\
\hline $2: 3$ & 2.277 & 2.047 \\
\hline $2: 2$ & 2.250 & 2.031 \\
\hline $2: 1$ & 1.915 & 1.741 \\
\hline $1: 3$ & 13.638 & 12.046 \\
\hline $1: 2$ & 10.150 & 9.072 \\
\hline $1: 1$ & 9.000 & 8.123 \\
\hline
\end{tabular}
an ion-penetrable charged membrane. In this case we have

TABLE 7

Variation in the Ratio $\left[n_{a, c, a ; b} / n_{a, c, 3: 3}\left(X_{0} \rightarrow \infty\right)\right]$ as a Function of $X_{0}$ and the Types of E lectrolyte Based on the D erjaguin A pproximation for $\Psi_{0} \rightarrow 0$ 


$$
n_{a, c}^{0}=\frac{\lambda \tanh ^{4}\left(a \psi_{d} / 4\right)}{a^{5}(a+b) k_{3}^{6}} \cdot \frac{\left(4 \pi \epsilon_{0} \epsilon_{r}\right)^{3}\left(k_{B} T\right)^{5}(48)^{2}}{e^{6} A_{123}^{2} \pi(\exp 2)}
$$

where $\psi_{d}$ is the dimensionless potential at the membraneliquid interface $(13,14)$. If the potential is high, Eq. [31] leads to Eq. [27]. On the other hand, if the potential is low, Eq. [31] reduces to Eq. [28], in general. Suppose that the fixed charges in a membrane phase arise from the exchange of cations in the liquid phase with the protons of the functional groups in membrane to form a complex, and the subsequent dissociation of this complex. If the binding of the cations with dissociated functional groups is sufficiently strong, and there are enough functional groups, then Eq. [31] reduces to Eq. [28], even if the potential is low (15).

A comparison between Tables 1 and 5, and 3 and 7 reveals that the CCC ratio of counterions obtained on the basis of a perturbation solution of the Poisson-Boltzmann equation and that based on the Derjaguin approximation are different appreciably. Since the derivation of the former is more rigorous than that of the latter, it is more reliable. Nevertheless, the Derjaguin approximation provides a quick estimation for the CCC ratio and can be used as an initial guess for the exact value.

In summary, the classic Schulze-Hardy rule is modified to the case of spherical particles immersed in an asymmetric electrolyte solution. We show that the CCC ratio of counterions is a function of both the valences of ion species and the sizes of the dispersed entities. To determine the CCC ratio of counterions for various combinations of electrolytes at a fixed particle size, the thickness of the Debye length needs to be taken into account. The present analysis provides an efficient method for the estimation of the CCC ratio of counterions.

\section{APPENDIX A}

Let $n_{a}$ and $n_{b}$ be the number concentrations of cation and anion, respectively. Then

$$
\rho=\mathrm{aen}_{a}-\mathrm{ben}_{b}
$$

Substituting this expression into Eq. [5] and employing the Boltzmann distributions for $n_{a}$ and $n_{b}$, we have

$$
\sigma=-\operatorname{aen}_{a}^{0} \int_{r_{0}}^{\infty}\left(e^{-a \psi}-e^{b \psi}\right)\left(\frac{r}{r_{0}}\right)^{2} d r
$$

Since the amount of coion near a charged surface is limited, we assume that it is negligible in the region $\left(r_{0}, r_{0}+\delta / \kappa\right)$, $\delta$ being a small number, and Eq. [A2] can be approximated by

$$
\sigma=\sigma_{u}+\sigma_{v}
$$

where

$$
\begin{gathered}
\sigma_{u}=-a e n_{a}^{0} \int_{r_{0}}^{r_{0}+\kappa^{-1} \delta} e^{-a \psi}\left(\frac{r}{r_{0}}\right)^{2} d r \\
\sigma_{v}=-\left.a(a+b) e n_{a}^{0} \kappa^{-1}\left(\frac{d \psi}{d X}\right)\right|_{X_{0}+\delta}
\end{gathered}
$$

Equation [A3b] can be calculated by integrating the Poisson-Boltzmann equation in the region $\left(r_{0}+\delta / \kappa, \infty\right)$, in which the potential is relatively low $(8,9)$. Substituting Eqs. [2]-[2c] into Eq. [A3a] and collecting terms of the order $X_{0}$ as $\psi_{0} \rightarrow-\infty$, we obtain

$$
\begin{aligned}
\sigma_{u} \cong \frac{-2 a e n_{a}^{0}}{k_{3} \kappa} & \left\{\int_{0}^{k_{3} \delta / 2} \tanh ^{2} Z d Z+\frac{2}{k_{3} X_{0}} \int_{0}^{k_{3} \delta / 2} \tanh ^{2} Z\right. \\
\times & {\left.\left[Z-\frac{e^{-2 Z}}{1-e^{-4 Z}}\left(1-2 Z-e^{-2 Z}\right)\right] d Z\right\} }
\end{aligned}
$$

where

$$
Z=k_{3}\left(X-X_{0}\right) / 2
$$

If $\delta$ is proportional to $L_{c}, k_{3} \delta / 2$ is roughly a constant. The value of $\sigma_{v}$ can be evaluated by substituting Eq. [2]-[2c] into Eq. [A3b]. We have, for $\psi_{0} \rightarrow-\infty$,

$$
\sigma_{v}=-4(a+b) e n_{a}^{0} \kappa^{-1}\left[k_{3}+\left(\frac{3}{2}-k_{3} \delta\right) / X_{0}\right] e^{-k_{3} \delta}
$$

For a strongly charged surface, the effect of curvature on surface charge density is negligible $(8,9)$, and $\sigma_{u} \gg \sigma_{v}$. We conclude that

$$
\sigma \cong \sigma_{u} \propto a n_{a}^{0} / k_{3} \kappa, \text { as } \psi_{0} \rightarrow-\infty
$$

On the other hand, if $\psi_{0} \rightarrow 0$, the surface charge density is described by Eq. [A3b] with $\delta=0$. Since $\tanh \left(a \psi_{0} / 4\right) \rightarrow$ $a \psi_{0} / 4$, substituting Eqs. [2]-[2c] into Eq. [A3b] leads to Eq. [21].

\section{APPENDIX B}

For a fixed particle size,

$$
X_{0} \propto \kappa \propto\left[a(a+b) n_{a}^{0}\right]^{1 / 2}
$$

On the basis of this expression, the ratio of $X_{0}$ for various 
combinations of $a: b$ electrolytes can be obtained. For example, the effect of counterions can be summarized as follows:

Type $S$ ratio

$$
X_{0,3: 3}: X_{0,2: 2}: X_{0,1: 1}=1:\left(\frac{4 n_{a, c, 2: 2}^{0}}{9 n_{a, c, 3: 3}^{0}}\right)^{1 / 2}:\left(\frac{n_{a, c, 1: 1}^{0}}{9 n_{a, c, 3: 3}^{0}}\right)^{1 / 2}
$$

Type $A_{1}$ ratio

$$
X_{0,3: 1}: X_{0,2: 1}: X_{0,1: 1}=1:\left(\frac{n_{a, c, 2: 1}^{0}}{2 n_{a, c, 3: 1}^{0}}\right)^{1 / 2}:\left(\frac{n_{a, c, 1: 1}^{0}}{6 n_{a, c, 3: 1}^{0}}\right)^{1 / 2}
$$

Type $A_{2}$ ratio

$$
X_{0,3: 2}: X_{0,2: 2}: X_{0,1: 2}=1:\left(\frac{8 n_{a, c, 2: 2}^{0}}{15 n_{a, c, 3: 2}^{0}}\right)^{1 / 2}:\left(\frac{n_{a, c, 1: 2}^{0}}{5 n_{a, c, 3: 2}^{0}}\right)^{1 / 2}
$$

Type $\mathrm{A}_{3}$ ratio

$$
X_{0,3: 3}: X_{0,2: 3}: X_{0,1: 3}=1:\left(\frac{5 n_{a, c, 2: 3}^{0}}{9 n_{a, c, 3: 3}^{0}}\right)^{1 / 2}:\left(\frac{2 n_{a, c, 1: 3}^{0}}{9 n_{a, c, 3: 3}^{0}}\right)^{1 / 2}
$$

In these expressions, $X_{0, a: b}$ is the value of $X_{0}$ for an $a: b$ electrolyte, type $S$ stands for symmetric electrolytes, and type $A_{b}, b=1,2,3$, stands for asymmetric electrolytes with common anion valence $b$. In general, the CCC ratio of counterions needs to be estimated through an iterative procedure. We suggest the following algorithm:

Step 1. Arbitrarily assume an $X_{0}$ ratio.

Step 2. The CCC ratio is calculated by reading values of $n_{a, c, a: b}^{0} / n_{a, c, 3: 3}^{0}\left(X_{0} \rightarrow \infty\right)$ from Table 1 for various $a: b$ combinations. Interpolations and extrapolations may be necessary at this stage.

Step 3. Calculate the $X_{0}$ ratio by Eqs. [B1a]-[B1d] and back to step 1 .

The iteration procedure is continued until the $X_{0}$ ratio converges. The corresponding ratio for $n_{a, c}^{0}$ is the desired CCC ratio of counterions.

Note that if $X_{0}$ is sufficiently large, the entry of Table 1 is a weak function of $X_{0}$. In this case, one iteration of the preceding algorithm yields satisfactory result. As an example for the present theory, we assume

$$
X_{0,3: 1}: X_{0,2: 1}: X_{0,1: 1}=20: 50: 200
$$

The corresponding entries in Table 1 lead to

$$
n_{a, c, 3: 1}^{0}: n_{a, c, 2: 1}^{0}: n_{a, c, 1: 1}^{0}=0.618: 9.336: 729
$$

Substituting these figures into Eq. [B1b] gives

$$
X_{0,3: 1}: X_{0,2: 1}: X_{0,1: 1}=20: 56: 229
$$

which is close to Eq. [B2a].

As an example for the result derived from the Derjaguin approximation, we assume

$$
X_{0,3: 1}: X_{0,2: 1}: X_{0,1: 1}=10: 25: \infty
$$

The corresponding entries in Table 5 lead to

$$
n_{a, c, 3: 1}^{0}: n_{a, c, 2: 1}^{0}: n_{a, c, 1: 1}^{0}=1: 15.8: 784
$$

Substituting these figures into Eq. [B1b] gives

$$
X_{0,3: 1}: X_{0,2: 1}: X_{0,1: 1}=10: 27.48: 140.21
$$

which is close to Eq. [B3a].

\section{REFERENCES}

1. Hunter, R. J., “Foundations of Colloid Science,', Vol. I. Oxford University Press, London, 1989.

2. Overbeek, J. Th. G., Pure and Appl. Chem. 52, 1151 (1980).

3. Wiese, G. R., and Healy, T. W., Trans. Faraday Soc. 66, 490 (1970).

4. Matijevic, E., Janauer, G. E., and Kerker, M., J. Colloid Sci. 19, 333 (1964).

5. Brenner, S. L., and Roberts, R. E., J. Phys. Chem. 77, 2367 (1973).

6. van Aken, G. A., Lekkerkerker, H. N. W., Overbeek, J. Th. G., and de Bruyn, P. L., J. Phys. Chem. 94, 8468 (1990).

7. Hsu, J. P., and Kuo, Y. C., J. Chem. Soc. Faraday Trans. 89, 1229 (1993).

8. Hsu, J. P., and Kuo, Y. C., J. Colloid Interface Sci. 167, 35 (1994).

9. Hsu, J. P., and Kuo, Y. C., J. Colloid Interface Sci. 170, 220 (1995).

10. Hsu, J. P., and Kuo, Y. C., J. Colloid Interface Sci. 171, 254 (1995).

11. Hsu, J. P., and Kuo, Y. C., J. Chem. Soc. Faraday Trans. 91, 1223 (1995).

12. Hiemenz, P. C., 'Principles of Colloid and Surface Chemistry.' Marcel Dekker, New York, 1977.

13. Hsu, J. P., and Kuo, Y. C., J. Colloid Interface Sci. 166, 208 (1994).

14. Hsu, J. P., and Kuo, Y. C., J. Colloid Interface Sci. 171, 483 (1995).

15. Hsu, J. P., and Kuo, Y. C., J. Colloid Interface Sci. 174, 250 (1995). 